\title{
Ciudades seguras y sin violencias para las mujeres y las niñas, avances y retos de la ciudad de Medellín, Colombia ${ }^{1}$
}

\section{SAFE CITIES SAFE AND WITHOUT VIOLENCE FOR WOMEN AND GIRLS, ADVANCES AND CHALLENGES OF THE CITY OF MEDELLÍN, COLOMBIA}

CIDADES SEGURAS E SEM VIOLÊNCIA PARA AS MULHERES E MENINAS, AVANÇOS E DESAFIOS DA CIDADE DE MEDELLÍN, COLÔMBIA

ANA MILENA Montoya Ruiz ${ }^{2}$

ÁNGELA MARÍA Correa Londoño ${ }^{3}$

Montoya Ruiz, A. M. y Correa Londoño, A. M. (2018). Ciudades seguras y sin violencias para las mujeres y las niñas, avances y retos de la ciudad de Medellín, Colombia. Perspectiva Geográfica, 23(2). 10.19053/01233769.7384

\section{Recepción:}

16 de octubre de 2017

Evaluación:

27 de abril de 2018

Aprobación:

30 de julio de 2018

\section{Resumen}

El siguiente artículo de investigación expone los resultados del Estudio exploratorio de la ciudad de Medellín para la implementación del Programa Ciudades Seguras Sin Violencia Hacia las Mujeres y Niñas Safe Cities y referentes explicativos para comprender las condiciones de inseguridad de las mujeres en los contextos urbanos.

1 Artículo de Investigación resultado del Estudio exploratorio realizado durante el segundo semestre de 2015 y primer semestre de 2016 en la ciudad de Medellín para la implementación del Programa Ciudades Seguras para las Mujeres y las Niñas Safe Cities, fases uno y dos, financiado por ONU Mujeres y la Secretaría de las Mujeres de la Alcaldía de Medellín.

2 Abogada de la Universidad de Medellín, Colombia. Candidata a doctora en Ciencias Humanas y Sociales de la Universidad Nacional, Sede Medellín. Magister en Género, Sociedad y Política del Programa Regional de Formación en Género y Políticas Públicas PRIGEPP-FLACSO Argentina. Docente e investigadora del Grupo de Investigaciones Jurídicas. Coordinadora de la Clínica Jurídica en Género y Derechos Humanos de la Facultad de Derecho de la Universidad de Medellín. Correo electrónico: amilemr@gmail.com, ammmontoyar@udem.edu.co 
Se realizó un recorrido por las políticas públicas de la ciudad de Medellín y por las estrategias implementadas en el mundo en el marco de Safe Cities para mejorar la seguridad en las urbes, que fortalecen las estrategias de seguridad pública para el logro de la equidad de género en el territorio.

Palabras claves: ciudades seguras para las mujeres, derecho a la ciudad, perspectiva de género, violencia basada en género, violencia urbana.

\section{Abstract}

The following research article exposes the results of the exploratory study of the city of Medellin for the implementation of the program cities safe without violence against women and girls Safe Cities and related explanatory to understand the unsafe conditions of women in urban contexts. It takes a tour by the policies of the city of Medellin and the strategies implemented in the framework of Safe Cities worldwide to improve security in cities, to strengthen the strategies of public security for the achievement of gender equality in the territory.

Keywords: safe cities for women, right to the city, gender, urban violence and gender-based violence.

\section{Resumo}

$\mathrm{O}$ artigo de pesquisa a seguir apresenta os resultados do estudo exploratório da cidade de Medellín para a implementação das cidades programa seguras sem violência contra mulheres e meninas cidades de cofre e explicativos relacionados para entender o condições inseguras de mulheres em contextos urbanos. É preciso um tour pelas políticas da cidade de Medellín e as estratégias implementadas no âmbito do seguro cidades em todo o mundo para melhorar a segurança nas cidades, para reforçar as estratégias de segurança pública para a consecução da igualdade de género no território.

Palavras-chave: cidades seguras para as mulheres, direito à cidade, sexo, violência urbana e violência baseada em gênero. 


\section{Introducción}

La violencia contra las mujeres es un problema cultural, político, económico y social en el mundo, que afecta el goce efectivo y la garantía de los derechos humanos de más de la mitad de la población global (ONU Mujeres, 2013). Entre sus causas se encuentran las desigualdades históricas y estructurales, tales como la división del espacio público y privado que las mujeres han enfrentado con relación a los hombres, quienes han impuesto un orden social orientado por los valores y prácticas de exclusión, subordinación y discriminación respecto a ellas, en escenarios de interacción social tales como el hogar, la calle, los sitios de trabajo, los espacios de decisión política o los lugares para el ocio y el esparcimiento, limitando el pleno disfrute de sus derechos y su configuración como sujetos autónomos y con capacidad de agenciamiento social y político.

Los movimientos feministas y sociales de mujeres, la reflexión académica, las redes de encuentro feminista y la institucionalidad de género a nivel internacional y local, particularmente la ciudad de Medellín, Colombia, preocupados por las diversas formas en las que se manifiesta y perpetúa la violencia contra las mujeres en los espacios privados y en los espacios públicos, y particularmente en estos últimos, han propuesto reflexiones sobre los intercambios que tienen lugar en la calle, el vecindario, la cuadra, la esquina, el parque o la plaza, y acciones para evitar las violencias de género en estos lugares porque en ellos se construyen y se llevan a cabo las prácticas sociales de relacionamiento entre hombres y mujeres, donde se imponen códigos y conductas que condicionan su uso, apropiación y ocupación (Red Mujer y Hábitat de América Latina, 2011).
El feminismo, como teoría social crítica y como movimiento social en pro del reconocimiento de los derechos humanos de las mujeres, ha propuesto nuevas categorías explicativas, como la existencia de un sistema sexo/género (Rubin, 1986) y la conceptualización de la categoría de género (Scott, 1986), entre otros recursos teóricos para comprender la realidad de las mujeres. Como producto de los aportes conceptuales del movimiento feminista y de sus desarrollos teóricos se ha identificado la existencia de relaciones sociosimbólicas entre hombres y mujeres que no son determinadas exclusivamente por condiciones biológicas y anatómicas, sino por procesos históricos y culturales. Estos aportes fueron el comienzo de una reflexión dinámica y crítica, que aún permanece, sobre la definición de las categorías de hombre y mujer, su época, el contexto y el lugar donde interactúan, y sobre la construcción de relaciones de poder y opresión que se encuentran presentes y se perpetúan en interacciones cotidianas, produciendo la violencia y la dominación sexual (Mackinnon, 1995) que se expresan con particularidades propias en ámbitos rurales y urbanos.

En ese sentido, la mirada de las mujeres y del feminismo ha generado nuevas comprensiones respecto a diversos aspectos del mundo social, entre los cuales se destacan sus preocupaciones sobre las manifestaciones de la violencia contra las mujeres y los lugares donde estas ocurren, el espacio público y privado y los espacios rurales y urbanos, estos últimos como centro de las relaciones entre mujeres y hombres en la modernidad y en la contemporaneidad. De ahí que las violencias contra las mujeres no pueden ser entendidas exclusivamente como violencias domésticas e intrafamiliares (Montoya Ruiz y Sierra Camargo, 2013), sino que se prolongan o se transfiguran en los espacios públicos. Es así 
como el lente del feminismo hace evidente que las ciudades no son construcciones sociales neutras al género, sino que son escenarios de disputa entre hombres y mujeres, quienes socializan y se apropian de formas diferenciales del espacio urbano e imponen normas sobre su ocupación, su uso, la propiedad de la vivienda, el aprovechamiento del suelo, las condiciones de infraestructura, la apropiación de los equipamientos urbanos, el uso de los medios de transporte y el goce de condiciones de seguridad personal y ciudadana. Estas disputas, a su vez, fueron identificadas en la ciudad de Medellín durante la realización del Estudio exploratorio de la ciudad de Medellín para la implementación del Programa Ciudades Seguras Sin Violencia Hacia las Mujeres y Niñas Safe Cities de ONU Mujeres, realizado entre 2015-2016, financiado por ONU Mujeres y la Secretaría de las Mujeres de la Alcaldía de Medellín.

La existencia de condiciones genéricas respecto al dominio del género masculino sobre el espacio público produce condiciones de violencia contra las mujeres, que se expresan de manera diferente a aquellas que acontecen en espacios privados, entre las que se encuentra el abuso sexual callejero y su mayor vulnerabilidad como víctimas de delitos de hurto. Estas diferencias se explican por la noción social de lo femenino y, en consecuencia, el lugar social que se les ha asignado a las mujeres, quienes en el escenario urbano enfrentan abusos de poder producto del ejercicio de una masculinidad hegemónica, que se traduce en prácticas androcéntricas de relacionamiento e interacción social en oposición a las mujeres.

El Estudio exploratorio de la ciudad de Medellín para la implementación del Programa Ciudades Seguras Sin Violencia Hacia las Mujeres y Niñas Safe Cities de ONU Mujeres, proyecto de investigación del cual se deriva este artículo, correspondió a la primera etapa de la implementación del programa Ciudades Seguras en el Medellín, en el cual se priorizó la Comuna 3 Manrique. Posterior a su realización fueron adelantadas otras investigaciones, como los criterios de construcción de la línea de base y el diseño de su fase de implementación en 2017. Además, se han realizado en la ciudad acciones posteriores en el marco de la política de seguridad pública para las mujeres de la ciudad de Medellín, su programa "Medellín segura para las mujeres y las niñas" y el proyecto "Territorios seguros para las mujeres y las niñas", que buscan generar condiciones para que ellas disfruten de espacios seguros en las comunas y los corregimientos y ejerzan libremente su ciudadanía, actualmente incorporados en el Plan de Desarrollo "Medellín cuenta con vos" 2016-2019. También hay otras acciones asociadas al proyecto de "Prevención y atención a las violencias basadas en género", como la Línea 123 Mujer, articulada a las agencias de seguridad de la administración local, o el programa de hogares de acogida para brindar protección temporal y acompañamiento jurídico y psicosocial a las mujeres víctimas de las violencias de género y a sus hijos e hijas.

Como antecedentes del Programa Ciudades Seguras Sin Violencia Hacia las Mujeres y Niñas Safe Cities de ONU Mujeres, está el Foro Urbano Mundial en la ciudad, en 2014, y la realización del recorrido exploratorio del barrio Moravia con la Red Mujer y Hábitat. En 2015, el ingreso de la ciudad de Medellín al Programa Global de Ciudades Seguras para las Mujeres y las Niñas, a través de carta de entendimiento entre la representante de ONU Mujeres, Belén Sanz, y el alcalde de Medellín para el período 2012-2015, Aníbal Gaviria Correa. La realización en 2016 del estudio exploratorio en la Comuna 3 
Manrique, la construcción del marco lógico para la implementación del programa, la definición de criterios para línea de base de la Comuna 3 y la elaboración del informe parcial sobre percepciones sobre el acoso y la violencia sexual de mujeres y niñas en el espacio público. Finalmente, en 2017 se destaca la construcción de la línea de base de la Comuna 3 y el informe final sobre percepción sobre el acoso y la violencia sexual de mujeres y niñas en el espacio público. Durante su ejecución, el gobierno local, de acuerdo a sus particularidades, problemáticas y circunstancias de tiempo, modo y lugar, ha priorizado las estrategias y la incorporación en los planes de desarrollo, y ha precisado la asignación de recursos pertinentes.

Durante el estudio exploratorio se identificaron algunas de las violencias urbanas, en concreto la violencia sexual en el espacio público, violencias que además de corresponder a un orden estructural sociosimbólico limitan a las mujeres el pleno ejercicio de sus derechos humanos, el goce de su derecho a la ciudad y el disfrute de los espacios urbanos y los bienes que la ciudad les ofrece (Montoya Ruiz y Builes Ceballos, 2015a, 2015b). La realización del Programa Ciudades Seguras en Medellín es una posibilidad para la construcción de contextos urbanos seguros y agradables para las mujeres de la ciudad y el goce de las posibilidades que las urbes ofrecen. Dicho programa ha dispuesto de varias metodologías para dar cuenta de la violencia contra las mujeres en el espacio público, identificar sus causas, realizar análisis sobre su ocurrencia y diseñar mecanismos técnico-administrativos para promover, ejecutar y monitorear el impacto de sus acciones. Entre estas se destacan, como herramientas metodológicas para la implementación del programa, las caminatas exploratorias, la construcción de sistemas de georreferenciación sobre los lugares inseguros en el territorio y los estudios exploratorios, los diagnósticos para la formulación del proyecto e implementación del programa, las actas de acuerdo y compromisos institucionales para la transformación urbanística y la conformación de los consejos locales de seguridad de las mujeres. Por último están los estudios exploratorios, una herramienta utilizada en este caso en particular.

Este artículo, en primer lugar, presenta los hallazgos del estudio exploratorio realizado en el contexto de la Comuna 3 Manrique de la ciudad de Medellín acerca de las violencias contra las mujeres en el espacio urbano. En segundo lugar, da cuenta de los logros de la institucionalidad de Medellín para construir la ciudad como un lugar seguro para las mujeres y las niñas, tales como las estrategias de seguridad pública para las mujeres y el logro de la equidad de género en el territorio, políticas que definen un marco institucional propicio para avanzar en la implementación del Programa de Ciudades Seguras en la ciudad de Medellín. En tercer lugar, realiza un recuento del Programa Safe Cities, promovido desde 2010 por ONU Mujeres en el mundo, con énfasis en las estrategias globales implementadas en diversos lugares para la inclusión de la dimensión de género en las políticas de seguridad pública, recorrido que aporta ideas para mejorar la seguridad de las mujeres en los espacios públicos de las ciudades y en Medellín. Por último, destaca los aportes y las discusiones promovidas por la lucha de las mujeres para la transformación de las ciudades. Finalmente, se concluye que las violencias contra las mujeres en los espacios públicos en las ciudades, particularmente en el contexto urbano de la ciudad de Medellín, tienen múltiples formas de expresión, por lo que no en pocos casos son aceptadas o naturalizadas como formas del relacionamiento urbano, por lo que se precisa de una política clara y acciones concretas 
de las mujeres para exigir que se les garantice una vida libre de violencias para las mujeres y las niñas.

\section{Metodología}

El goce y el disfrute diferencial del espacio público que realizan hombres y mujeres, así como las imposibilidades y temores que encuentran en ellos, aumentan sus percepciones y sensaciones de inseguridad y limitan el ejercicio de una ciudadanía femenina plena. La investigación realizada fue un estudio exploratorio, cuyo propósito fue aproximarse a las condiciones de inseguridad de las mujeres en el contexto de la Comuna 3 Manrique de la ciudad de Medellín, seleccionada por los altos índices de violencias sexuales reportados en 2014 y 2015, y conocer las acciones implementadas por la institucionalidad para el fortalecimiento de las estrategias de seguridad pública. Durante su desarrollo fueron realizadas visitas y entrevistas a funcionarios de la administración municipal y organizaciones sociales incidentes en la comuna o en la ciudad por su trabajo con mujeres; además, se realizaron cinco grupos focales con hombres y mujeres, adultos y jóvenes de la comuna, en los que se ahondó acerca de las violencias contra las mujeres en los espacios públicos de la ciudad y se identificaron los principales problemas que afectan las condiciones de seguridad de las mujeres en la ciudad. Finalmente, se indagó por las acciones implementadas en el mundo para mejorar la seguridad pública de las mujeres en los espacios públicos y en los medios de transporte, tendientes a erradicar y prevenir las violencias contra las mujeres en los contextos urbanos.

En términos metodológicos, los resultados que se presentan en este artículo son el producto de una investigación exploratoria, la cual es el primer paso para examinar un tema o problema poco estudiado y aumentar el grado de familiaridad con los lugares y con los fenómenos que allí ocurren. Este tipo de investigaciones sirven para "obtener información sobre la posibilidad de llevar a cabo una investigación más completa sobre un contexto particular de la vida real, investigar problemas del comportamiento humano que consideren cruciales profesionales de determinada área, identificar conceptos o variables promisorias, establecer prioridades para investigaciones posteriores o sugerir afirmaciones" (Hernández Sampieri, Fernández Collado y Baptista Lucio, 1991, p. 60). El estudio exploratorio en cuestión indagó por las distintas expresiones de la violencia contra las mujeres en el espacio urbano y público, entre ellas el acoso sexual callejero, las violaciones en espacios públicos o la ocurrencia de hurtos a mujeres, hechos violentos que inciden sobre la manera como ellas se sienten y perciben la inseguridad urbana.

El enfoque epistemológico que orientó el trabajo es el de investigación feminista, en tanto intentó recuperar el punto de vista de las mujeres para construir un panorama sobre la violencia urbana en la ciudad de Medellín. En su desarrollo se valoró su experiencia personal y se consideró que la superación de la violencia de la cual son víctimas las mujeres en los espacios urbanos es necesaria para mejorar sus condiciones de vida y superar las situaciones de opresión y discriminación que las afectan. De esta manera, su punto de vista como mujeres proporcionó una óptica diferente para conocer la realidad social, la cual, según la epistemóloga feminista Sandra Harding (1996), otorga a las mujeres la condición de sujetos cognoscentes, donde intervienen la intuición y los afectos. Sus voces ofrecieron una perspectiva particular acerca del acoso sexual callejero y otras violencias producidas en los espacios públicos que 
las afectan directa y diferencialmente, para ofrecer su mirada sobre la construcción de las relaciones de género en el espacio público y los fenómenos en los cuales el género influye en las urbes. Su desarrollo implicó el acercamiento a distintas fuentes de recolección de información, tales como la revisión documental de experiencias en el mundo para mejorar la seguridad de las mujeres en la ciudad, el análisis de documentos públicos producidos por la Secretaría de las Mujeres de Medellín y la consideración de la voz de algunas mujeres de la ciudad para conocer sus sentimientos y percepciones acerca de la inseguridad y la violencia sexual callejera.

\section{Resultados de investigación}

La violencia contra las mujeres y las niñas es considerada una vulneración y negación de sus derechos humanos, situación que responde a un orden social androcéntrico cuyas consecuencias han sido denunciadas por los movimientos feministas y sociales de mujeres en el mundo. Sus demandas son las que han llevado a los Estados a reconocer este tipo de violencia y prevenir su ocurrencia en todos los ámbitos en que se presenta mediante la expedición de legislaciones, políticas públicas y acciones administrativas dirigidas a la protección de las mujeres, entre ellas la Convención sobre la Eliminación de todas las Formas de Discriminación contra la Mujer (CEDAW), aprobada en 1979 y vigente como tratado internacional en 1981 (Asamblea General de las Naciones Unidas, 1979), y su correlato en el ámbito interamericano, la Convención Interamericana para Prevenir, Sancionar y Erradicar la Violencia contra la Mujer, conocida como "Convención de Belém do Pará" (1994).
Las mujeres se encuentran expuestas a múltiples violencias tanto en los espacios privados como en los espacios públicos, y es en estos últimos donde se transforman las conductas violentas y se proyectan las consecuencias de la violencia doméstica. Para acercarnos a la identificación y la explicación de las violencias que afectan la vida de las mujeres en los centros urbanos, aceptamos la definición de violencia contra las mujeres presentada por la CEDAW, donde se define como "todo acto de violencia basado en la pertenencia al sexo femenino que tenga o pueda tener como resultado un daño o sufrimiento físico, sexual o sicológico para la mujer, así como las amenazas de tales actos, la coacción o la privación arbitraria de la libertad, tanto si se producen en la vida pública como en la vida privada" (Asamblea General de las Naciones Unidas, 1979), que produce daños o sufrimientos físicos, psicológicos, sexuales y económicos o patrimoniales. Por esto la violencia basada en género que sufren las mujeres en los espacios públicos y en las ciudades no pueden quedar al margen de los debates sobre las políticas urbanas, la violencia social y la seguridad e insegura en las ciudades (Red Mujer y Hábitat de América Latina, 2011).

\subsection{La violencia contra las mujeres y las niñas en la ciudad de Medellín}

Las situaciones de inseguridad en los espacios públicos y privados limitan la incidencia de las mujeres en la vida comunitaria y el pleno disfrute de oportunidades laborales, educativas y de ocio, e incluso llegan a restringirles los bienes y servicios que la ciudad tiene para ofrecerles. Según la Encuesta de Nacional de Calidad de Vida de 2014 (Departamento Administrativo Nacional de Estadística, 2014), Medellín cuenta con 2.441 .123 habitantes, de los cuales 1.148.961, equivalentes al 47,07\%, 
son hombres, y 1.292.162, equivalentes al 52,93\%, son mujeres, población que se encuentra ubicada en 16 comunas y cinco corregimientos en los que ocurren situaciones delictivas en espacios públicos y privados que vinculan y afectan diferencialmente a hombres y mujeres, entre los que se destacan homicidios, hurtos a residencias, hurtos callejeros, hurtos en motocicletas, hurtos de vehículos, hurtos a establecimientos públicos y extorsiones. Las mujeres son las principales víctimas del hurto callejero consumado a través de atracos por factor de oportunidad, halado, violación de cerraduras, cosquilleo y raponazo, en medios de transporte masivo y transporte informal, conductas que son poco denunciadas por su insuficiencia probatoria.

Por su parte, en informes de la Secretaría de las Mujeres, en el período 2012-2015 existe un incremento de delitos con víctimas mujeres: son el 88\% de las víctimas de delitos sexuales, el $83 \%$ de las víctimas de violencia intrafamiliar y el $40 \%$ de las víctimas de homicidios, los cuales, en su mayoría, se corresponden con el feminicidio. Situaciones que se agravan en la Comuna 3 de la ciudad de Medellín, ubicada en la zona nororiental, que para la época del estudio se encuentra en el quinto lugar de poblamiento urbano de la ciudad, con una tasa de crecimiento del 5,8\% en los últimos ocho años, equivalentes a 311 habitantes por hectárea para el 2014 que la convierte en un territorio densamente poblado, donde se asienta clases sociales medias y bajas de la ciudad. Para la fecha del estudio, el número total de sus pobladores era 159.658 habitantes, de los cuales 83.148 eran mujeres (52,08\%) y 76.510 eran hombres $(47,92 \%)$, por lo cual su población femenina corresponde al $6,41 \%$ de las mujeres de Medellín (Montoya Ruiz y Builes Ceballos, 2015a, 2015b). El crecimiento poblacional y la comisión de delitos inciden en el análisis sobre las formas diferenciales en que las mujeres y los hombres disfrutan de la vida urbana, lo que convierte sus condiciones de seguridad en un tema de especial atención por parte de las administraciones públicas, que deben actuar ante el aumento de las violencias contra las mujeres en los espacios públicos y en las calles.

Otro factor influyente en el aumento de las violencias contra las mujeres en los espacios públicos en Medellín son las altas tasas de violencia intrafamiliar. En 2016 la ciudad ocupó el segundo lugar en casos de ocurrencia de violencia intrafamiliar en Colombia; en total hubo 7.327 reportes y el $88 \%$ de sus víctimas fueron mujeres (Secretaría de Seguridad y Convivencia, 2017). Sus causas no pueden ser comprendidas exclusivamente desde los contextos privados, toda vez que esta afecta desfavorablemente la construcción de vínculos sociales, una vez se admite la interrelación entre familia y sociedad, pero, además, la violencia intrafamiliar (en adelante, VIF) pone en riesgo una de las aspiraciones más valiosas en la sociedad contemporánea, esto es, la constitución de las mujeres como sujetos de derecho, dados los efectos personales, familiares, sociales y culturales que desencadena dicha expresión de la violencia (Corporación para la Vida Mujeres que Crean y Corporación Vamos Mujer, 2015). 


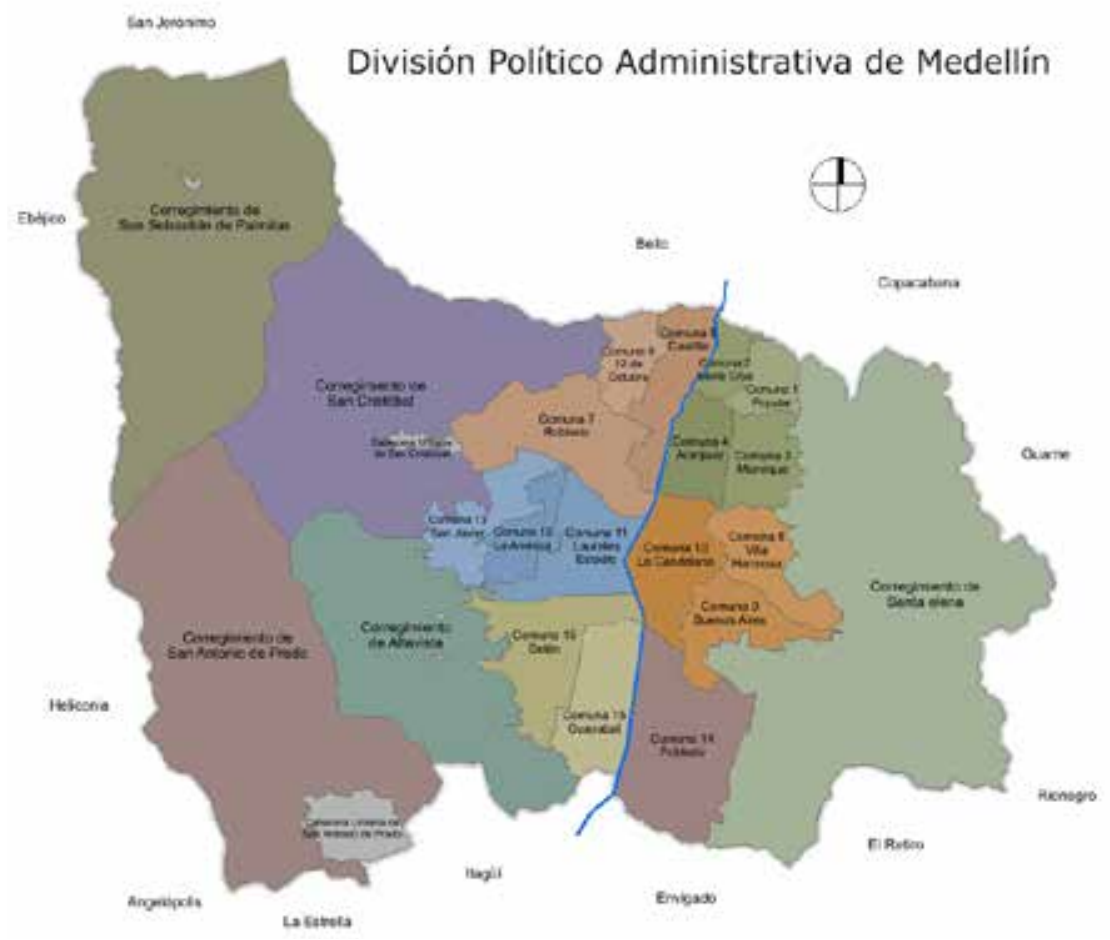

FIGURA 1.

Organización administrativa y territorial de la ciudad de Medellín.

Fuente: Commons Wikipedia.
Las mujeres, además de ser víctimas de delitos comunes y violencia intrafamiliar en la Comuna 3 , han sido víctimas de la violencia sexual en espacios públicos, que no denuncian por temor, por impedimentos para acudir a la ruta de atención, por presiones para no denunciar o por la aceptación en la comunidad de la justicia por propia mano, ejercida a través del accionar de los "combos" de los distintos sectores, a quienes acuden las mujeres por la ineficiencia de instituciones como la Policía Nacional y la tardanza de las rutas de atención. Por otra parte, la existencia de normas de comportamiento social con las que se imponen estereotipos a las mujeres invisibilizan la violencia sexual contra ellas en los espacios públicos, naturalizando los silbidos, las miradas lascivas, las propuestas sexuales, los comentarios indecentes, la obstrucción de la circulación, la persecución y la toma de fotografías y videos sin consentimiento (Montoya Ruiz y Builes Ceballos, 2015a, 2015b).
De ahí que los espacios influyen en las formas como se configuran las violencias, entendidas como un fenómeno multidimensional que convierte problemáticas locales en asuntos globales, como lo señala Mónica Colombara (2011) al analizar las relaciones entre violencia urbana y violencia de género: "la violencia tiene múltiples actores, directos e indirectos, es cambiante en la historia y en el espacio"(p. 4). Algunos de los factores que influyen en perpetuación de violencias urbanas y de género en las ciudades se encuentran asociados con el transporte, los servicios e infraestructuras inadecuadas, e inclusive inexistentes, y las dificultades de inaccesibilidad al suelo urbano, la carencia de vivienda, el deterioro ambiental o los problemas de gobernabilidad. Dichas problemáticas responden a lógicas urbanistas que privilegian la segregación residencial, los sentimientos de miedo, inseguridad, angustia y desamparo, y contribuyen a formas de interacción social entre los géneros que resultan 
violentas, discriminatorias e intolerantes y producen una estrecha relación entre violencia urbana, ciudad, modelos de desarrollo urbano y violencia basada en género.

Por otra parte, la percepción de inseguridad que tienen las mujeres, según los grupos focales realizados, se encuentra determinada por sus sentimientos de temor y por las condiciones objetivas del peligro que les representa el disfrute de la vida nocturna. Entre ellos se encuentran tanto los peligros asociados al consumo de drogas y alcohol como al aumento del riesgo de violencia sexual hacia mujeres, adolescentes y niñas, que propician el ejercicio de la prostitución como fuente de ingresos y la existencia de redes de explotación y abuso sexual y facilitan la comisión del delito de trata de personas, el pago de favores sexuales, los riesgos en su salud por la ingesta de bebidas alcohólicas y el consumo voluntario e involuntario de drogas. Adicionalmente, se encuentran los peligros asociados al hacinamiento y las dificultades de acceso a los medios de transporte formal, que contribuyen a incrementar las rutas informales en los barrios de la ciudad y aumentan su vulnerabilidad. Así mismo, existen temores al recorrer espacios públicos como parques, canchas, gimnasios al aire libre, unidades deportivas o las afueras de instituciones educativas producidos por la alta presencia masculina, la escasez de iluminación o la poca concurrencia de personas que les permita sentirse seguras, situaciones que condicionan la existencia de rutinas y horarios que restringen su circulación.

Todo lo anterior se interrelaciona con la existencia de una masculinidad hegemónica en la ciudad que impone normas de comportamiento, tales como actos exhibicionistas, piropos vulgares, obstrucción de su circulación o miradas libidinosas que se consideran normales, o se excluye a las mujeres de ciertos espacios, como placas deportivas, bares, cafés y billares por ser espacios de socialización masculina; ambas situaciones inciden en la naturalización de la violencia sexual hacia las mujeres en los espacios públicos en Medellín (Montoya Ruiz y Builes Ceballos, 2015a, 2015b). Los estudios urbanos referidos a la seguridad e inseguridad en las ciudades han subestimado las violencias producidas por la asimetría de poder entre los géneros y las situaciones que atraviesan la vida cotidiana de las mujeres, asunto que ha sido analizado por el feminismo (Del Valle, 1997). Ante los logros del feminismo, como el reconocimiento de derechos a las mujeres y el alcance de su autonomía y empoderamiento para acceder a la vida pública y a los espacios públicos, los centros urbanos se han convertido en epicentros de múltiples violencias producidas por las relaciones sociosimbólicas entre hombres y mujeres, las cuales se complejizan ante la permisividad que produce el anonimato en las urbes, la densidad demográfica o la escasez del enfoque de género en la planificación urbana. De tal forma, ciudades como Medellín y sus comunas son lugares donde se reproducen relaciones de poder inequitativas entre los géneros que se expresan mediante violencias físicas y simbólicas, que producen temor, y riesgos relacionados con el uso de determinados espacios, recorridos u horarios que condicionan los desplazamientos, los itinerarios y las prácticas de uso de los servicios de las urbes por parte de las mujeres, quienes se encuentran expuestas a las violencias ejercidas por desconocidos (Falú y Rainero, 1995).

Además, las violencias sociales, políticas, institucionales y económicas se espacializan en las ciudades; particularmente en Medellín la ocurrencia de las violencias refleja conexiones con el territorio y las formas de ejercicio del poder. En la ciudad, la confi- 
guración territorial se encuentra ligada a las formas de construcción de la masculinidad, aspecto que incide tanto en la organización espacial y la disposición de los servicios públicos como en la ocurrencia de conductas delictivas que afectan a las mujeres, tales como los abusos sexuales en el espacio público o la delincuencia callejera que las ponen en una situación de mayor vulnerabilidad. Así mismo, influye en expresiones de violencia institucional perpetrada por autoridades estatales o en expresiones de violencia política impulsadas por actores en pro de lograr o mantener el poder político, tales como grupos armados ilegales que han sometido sexualmente a las mujeres como una estrategia de guerra.

De acuerdo con los avances de la legislación internacional y nacional vigente, las violencias contra las mujeres ocurridas en ámbitos privados y públicos se han hecho particularmente visibles y pueden manifestarse mediante las situaciones presentes en el contexto de la ciudad y sus comunas que se detallan en la Tabla 1.

\section{TABLA 1. VIOLENCIAS CONTRA LAS MUJERES EN LAS CIUDADES}

TIPO DE VIOLENCIA BASADA EN GÉNERO

Violencia física: se produce por riesgo o disminución de la integridad corporal de una persona.

Violencia psicológica: se produce por la acción u omisión destinada a degradar o controlar las acciones, comportamientos, creencias y decisiones de otras personas.

Violencia sexual: consiste en la acción de obligar a una persona a mantener contacto sexualizado, físico o verbal, o a participar en otras interacciones sexuales mediante el uso de fuerza, intimidación, coerción, chantaje, soborno, manipulación, amenaza o cualquier otro mecanismo que anule o limite la voluntad personal.

Violencia económica y patrimonial: se relaciona con la pérdida, transformación, sustracción, destrucción, retención o extracción de objetos, instrumentos de trabajo, documentos personales, bienes, valores, derechos o atributos económicos destinados a satisfacer las necesidades de la mujer.

Violencia institucional: se incluye como una violencia de género y se manifiesta en la negligencia y la atención inadecuada por parte del Estado.

\section{MANIFESTACIÓN COMO VIOLENCIA URBANA CONTRA LAS MUJERES}

Asesinatos, atracos, robos, asaltos, inmovilización, estrangulamientos, golpes, patadas, empujones, mechoneadas, cachetadas, zarandeos, torceduras, persecución, forcejeos, abortos provocados por golpes, pisones, quemaduras, coscorrones y puños, entre otros.

Piropos morbosos, insultos, gritos, amenazas, comentarios y chistes sexistas, irrespeto, humillaciones, coacción, manipulación, descalificación, indiferencia, sarcasmo, desconocimiento, posesión, engaño, discriminación, falta de reconocimiento, intimidación o estereotipación.

Manoseo, violación, miradas lascivas, inducción a la prostitución, abuso sexual, incesto, violación conyugal, exhibicionismo, inducción al aborto, presión a la procreación, roces, utilización del cuerpo de la mujer como botín de guerra y ajuste de cuentas entre pandillas.

No reconocimiento de la economía del cuidado, detrimento y pérdida del patrimonio, despojo y daños de bienes, menores ingresos, oficios no calificados y desvalorados por su condición de mujer, explotación, triple jornada, inasistencia alimentaria, falta de oportunidades y garantías laborales, flexibilización de horarios, múltiples barreras para asumir cargos de poder, acoso laboral y labores comunitarias no remuneradas.

Negligencia, atención inadecuada por parte del Estado, revictimización, estigmatización, información inadecuada, desconocimiento de derechos, desinterés, irrespeto y falta de escucha por los agentes del Estado y la institucionalidad pública.

Fuente: elaboración propia. 
De ahí que la respuesta a las violencias ha sido la necesidad de seguridad, concepto que a su vez ha sido repensado por la reflexión feminista, pensamiento y acción, para el cual la erradicación de las violencias en las ciudades y la paz en ellas dependerán de la transformación de las calles, las aceras y las relaciones vecinales, y no de la existencia de controles y autoridades. Como fue propuesto por la urbanista y feminista Jane Jacobs (2011), "la paz pública de las ciudades - la paz en las calles y en las aceras - no tiene por qué garantizarse de manera esencial por la policía, por muy necesaria que ésta sea. Esa paz ha de garantizarla principalmente una densa y casi inconsciente red de controles y reflejos voluntarios y reforzada por la propia gente" (p. 58).

Por lo anterior el contexto de las violencias urbanas en la ciudad de Medellín y en su Comuna 3, donde existe un contínuum de violencias, es un reflejo de aquellas agresiones a las que las mujeres están expuestas en contextos caracterizados por altos índices de delitos, alta población masculina, bajos niveles de empleabilidad y altas tasas de violencia intrafamiliar. La concurrencia de estos factores genera condiciones de riesgo en las mujeres como sujetos pasivos de delitos tales como la violencia sexual y actos abusivos tolerados por la comunidad, como la violencia sexual callejera; estos últimos son desestimados por la comunidad porque son la forma natural de relacionamiento, razón por la cual las autoridades no los persiguen ni los investigan. En consecuencia, las violencias en los espacios privados aumentarán las violencias de género en los espacios públicos, producirán condiciones reales de inseguridad (dimensión objetiva) y aumentarán las percepciones sobre la seguridad y la inseguridad en los espacios urbanos (dimensión subjetiva); ambas dimensiones influyen en las construcciones sociales del género y en las formas diferenciales como los hombres y las mujeres usan y se apropian de los espacios.

\subsection{Fortalecimiento del marco institucional, avances para la implementación del Programa Safe Cites}

La ciudad de Medellín, desde la década de 1990, avanza en la configuración de una institucionalidad para la defensa, la promoción y la protección de los derechos humanos de las mujeres y promueve políticas públicas para el fortalecimiento y el agenciamiento de su ciudadanía femenina. En 1992 creó una institucionalidad de género en respuesta a los retos impuestos por la ratificación por parte del Estado colombiano de la Convención sobre la Eliminación de todas las Formas de Discriminación Contra la Mujer (CEDAW) y la activa movilización de los movimientos feministas y sociales de las mujeres, que han exigido la formulación de políticas públicas para la transformación de las condiciones materiales de su existencia. En 2003 la ciudad aprobó la Política Pública para las Mujeres Urbanas y Rurales (Acuerdo 22 de 2003) como instrumento de orientación, planificación y ejecución de programas y proyectos para el mejoramiento de la calidad de vida y la construcción de la ciudadanía plena de las mujeres (Concejo de Medellín, 2003, artículo 1). Entre sus propósitos se encuentra promover la educación, la salud, el empleo y los derechos humanos de las mujeres, así como consolidar sus procesos de empoderamiento, el ejercicio de la ciudadanía plena, el incremento de la gobernabilidad y la convivencia pacífica, y el fortalecimiento de la democracia incluyente y participativa, por ser condiciones para el desarrollo humano equitativo, sustentable y sostenible en la ciudad (Concejo de Medellín, 2003, artículo 4). 
En 2007 fue creada la Secretaría de las Mujeres como entidad adscrita al despacho del alcalde de la ciudad (Acuerdo Municipal 01 de 2007), con lo cual se ampliaron los alcances de la institucionalidad de género, que se proyecta como la entidad rectora de la política pública de las mujeres, con la misión de contribuir a la igualdad de derechos y oportunidades y la disminución de prácticas discriminatorias que atenten contra el desarrollo político, social, económico y cultural de las mujeres del municipio de Medellín, a través del diseño, la implementación, la coordinación, el monitoreo y las asesorías de las políticas, planes y programas de la Alcaldía Municipal (Concejo de Medellín, 2007, artículo 2). La institucionalidad de género de la ciudad ha promovido acciones tendientes al mejoramiento de la seguridad pública de las mujeres formulando planes estratégicos y prioritarios para atender las necesidades prácticas y los interés estratégicos de las mujeres de la ciudad, entre ellos, en 2011, el Programa de Protección Integral a Mujeres Víctimas de Violencias (Acuerdo 52 de 2011), que ofrece protección, apoyo, atención psicosocial, acompañamiento y representación jurídica, hogares de acogida, capacitación, emprendimiento, sistemas de información, prevención y orientación para la protección, reparación y estabilización de víctimas de todos los tipos de violencias (Concejo de Medellín, 2011). Para el 2015 se registran avances tales como el Programa de Seguridad Pública para las Mujeres, la participación en mesas y comités para su articulación, los centros de equidad y el proyecto de educación incluyente para las mujeres, desde el cual se ha trabajado en la prevención de las violencias de género, sexual, física e intrafamiliar. No obstante, en el período 2016-2019 algunos de estos programas se vieron afectados por las disminuciones presupuestales $\mathrm{y}$ la transformación de su enfoque.
De igual manera, hay logros significativos en la oferta institucional para la construcción de una vida libre de violencias para las mujeres urbanas y rurales. Desde 2008 se construyen y fortalecen diversas estrategias claves para articular el Programa Safe Cities, entre ellas la Estrategia de Seguridad Pública, que avanzó hasta convertirse en el Programa de Seguridad Pública para las Mujeres, en el marco del Plan de Desarrollo Municipal "Medellín, un hogar para la vida" 2012-2015, orientado al avance en la promoción de una vida segura y libre de violencias tanto en espacios privados como públicos, estrategia a la cual pertenece el Programa de Seguridad Pública para las Mujeres con el que se aporta a la construcción de contextos urbanos no violentos para las mujeres y la creación de un sistema de gestión de información para avanzar en el conocimiento sobre la situación de su seguridad. Adicionalmente, se fortaleció un sistema institucional para la seguridad urbana de las mujeres, al cual pertenece el Consejo de Seguridad Pública para las Mujeres de Medellín, y se promovieron otras estrategias, como los mecanismos sociales e institucionales para la protección, la atención y el empoderamiento de las mujeres víctimas, la atención psicológica y jurídica en el territorio, los grupos terapéuticos, la defensa técnica de mujeres víctimas de violencia, los circuitos sociales de advertencia de riesgos (CSAR), la Línea 123 Mujer o los hogares de acogida. También fue promovida una agenda pública de alianzas Estado-sociedad civil para desarrollar cátedras y diplomados en género y justicia y la escuela itinerante de paz, entre otros productos comunicativos y pedagógicos para sensibilizar sobre la violencia basada en género.

Otros antecedentes institucionales que constituyen logros para avanzar en la construcción de una ciudad segura para las mujeres fueron la implementación 
del Plan Integral de Seguridad y Convivencia de Medellín (Plan de Desarrollo Municipal 2012-2015, "Medellín, un hogar para la vida"), que promovió la gestión de la seguridad ciudadana con enfoque de derechos, de género y diferencial, así como la creación de un Sistema de Información y Conocimiento de Género en Medellín (SICGEM). Así mismo, está la incorporación del principio de equidad de género en el Plan de Ordenamiento Territorial con el propósito de identificar los impactos diferenciados de la planeación territorial en hombres y mujeres y formular propuestas para el desarrollo de una ciudad y una región equitativa desde el punto de vista social y de género, incluyente y sostenible. También están los debates desarrollados en el marco del $7^{\circ}$ Foro Urbano Mundial WUF7 en 2014, convocado por el Programa de Asentamientos Humanos de Naciones Unidas (ONU Hábitat), espacio que avanzó en la discusión global sobre el desarrollo urbano, la equidad y la justicia para hombres y mujeres en la ciudad. En este evento, la mesa de Género en Medellín fue un espacio clave para incluir en la agenda pública las violencias contra las mujeres en los espacios públicos y en sus vidas cotidianas, que motivó indagaciones sobre las percepciones de seguridad que tienen las mujeres respecto a los espacios urbanos, con miras al diseño de programas y acciones encaminados a disminuir y prevenir las violencias urbanas que inciden en el disfrute y el uso de la ciudad. Por último, en 2014-2015 se realizaron las auditorías de seguridad para las mujeres, las caminatas exploratorias, la georreferenciación de las violencias y la identificación de los espacios percibidos como inseguros en las comunas de la ciudad.

Estos sucesos fueron los antecedentes que facilitaron la inclusión de la ciudad de Medellín en el Programa Safe Cities de ONU Mujeres, así como su compromiso como ciudad para diseñar estrategias innovadoras para la superación de las violencias urbanas y las violencias sexuales callejeras, compromiso que aún se encuentra pendiente. La existencia de un marco normativo institucional y de políticas públicas favorables es importante pero no suficiente, puesto que aún es un tema aplazado en las agendas institucionales y un propósito del movimiento feminista de Medellín y del mundo el visibilizar las violencias de género en el espacio público y repudiar el acoso sexual callejero, conductas que devienen en un contínuum de violencias como el acoso y el abuso sexual, el acceso carnal violento y abusivo, los asaltos, la explotación sexual, la trata de personas y los feminicidios. Según informes de ONU Hábitat en 2016 y 2017, la inseguridad urbana corresponde, en primer lugar, a causas sociales atribuidas a situaciones de exclusión social debidas al desempleo y la marginación prolongada o el analfabetismo y las modificaciones estructurales de la familia que hacen de la violencia intrafamiliar una causa de la violencia en las calles. En segundo lugar se encuentran las causas institucionales, entre ellas la inadecuación del sistema de justicia y la ineficaz respuesta de las instituciones encargadas de garantizar la seguridad. Por último se encuentran las causas ligadas al entorno, tales como la urbanización incontrolada, el surgimiento masivo de espacios semipúblicos, como establecimientos de comercio abiertos al público, la ilegalidad de los barrios que se transforman en zonas bajo control de mafias locales, grupos armados y delincuencia común organizada en combos (ONU Hábitat, 2017).

De tal manera, serán las particularidades del contexto y las desigualdades estructurales las que conviertan en peligrosos los parques, las calles, los medios de transporte, los baños públicos, los sitios de distribución de alimentos, las placas de recreación y 
deporte, los caminos, los parajes o los vecindarios. Retomando el legado de la arquitecta y urbanista feminista Jane Jacobs (2011), problemáticas como estas hacen prioritario repensar la planificación de las ciudades puesto que los modelos existentes son insuficientes, ya que no consideran las realidades en las que vive la población. Con su mirada feminista sobre la ciudad nos propone "otra planificación" que tenga en cuenta la experiencia cotidiana y las necesidades de las personas, que no constriña o reprima sus vidas y considere urgente construir entornos seguros para aumentar la percepción de seguridad en el espacio público.

\subsection{Recuento de la implementación global del Programa Safe Cities promovido desde 2010 por ONU Mujeres}

La iniciativa Ciudades Seguras Libres de Violencia contra las Mujeres y Niñas Safe Cities fue promovida en 2010 por ONU Mujeres, ONU Hábitat, Mujeres y Ciudades Internacionales, la Comisión Huairou y la Red Mujer y Hábitat de América Latina y el Caribe entre otros socios mundiales y locales inspirados por el activismo feminista para mejorar la seguridad de las mujeres y las niñas en los espacios públicos. En el marco de dicho programa se han propuesto alternativas para superar los problemas de inseguridad urbana que padecen las mujeres en las ciudades, y entre sus logros más importantes se encuentra su aporte a la implementación y la evaluación de enfoques integrales para la prevención de la violencia sexual y otras violencias ocurridas en espacios públicos incidentes en los contextos urbanos de ciudades como Quito (Ecuador), El Cairo (Egipto), Nueva Delhi (India), Port Moresby (Papúa Nueva Guinea) y Kigali (Ruanda), San José (Costa Rica), Tegucigalpa (Honduras), Nairobi (Kenia),
Beirut (Líbano), Marrakech (Marruecos), Manila (Filipinas), Dusambé (Tayikistán) Río de Janeiro (Brasil), Ciudad de México (México), Dublín (Irlanda), Winnipeg (Canadá), Sakai (Japón), Ciudad del Cabo (Sudáfrica), Reikiavik (Islandia), Nueva York (Estados Unidos), Medellín (Colombia), Ciudad de México (México) y Seúl (Corea del Sur), entre otras $^{4}$ (ONU Mujeres, s. f.).

El principal objetivo de Ciudades Seguras ha sido desarrollar, probar y difundir modelos de actuación para prevenir y reducir la violencia contra las mujeres y las niñas en los espacios públicos y convertirlos en espacios seguros y de oportunidades para el disfrute de sus vidas y el goce efectivo de sus derechos. Para lograr este propósito se requiere del abordaje de las brechas a nivel político y práctico en las vidas de las mujeres, las cuales crean barreras reales que les impiden gozar y ejercer sus derechos humanos en los contextos urbanos. Se han implementado diversas estrategias orientadas a la transformación de las ciudades, como la realización de estudios exploratorios, la evaluación del impacto de programas encaminados a eliminar la violencia de género como estrategia para prevenir y reducir el acoso sexual de las mujeres y las niñas en los espacios públicos, y el diseño, la implementación y la evaluación de un modelo local para prevenir las violencias en las ciudades. Entre los resultados esperados con la implementación de estas iniciativas

4 Otras ciudades integrantes a nivel global son: Bruselas, Cairo, Ciudad de Cabo, Ciudad de México, Ciudad de Quezon, Dublín, Duchanbé, Kigali, Marrakech, Medellín, Metro Manila, Nueva Delhi, Nueva York, Port Moresby, Puebla, Quito, Rabat, Reykjavik, Río de Janeiro, Sakai, Tegucigalpa, Torreón y Winnipeg. En cada ciudad participan y cooperan las diferentes instancias de gobierno y poderes del Estado, las organizaciones de mujeres y jóvenes, las ONG y las organizaciones de la sociedad civil, la academia y los centros de investigación, el sector privado, los medios de comunicación, la ONU, y cada vez más mujeres y hombres se unen para poner fin a la violencia contra las mujeres y las niñas en el espacio público. 
se encuentran: 1) el incremento de la percepción de seguridad por parte de las mujeres en los espacios públicos, así como un mayor uso de estos; 2) el aumento en la movilidad de las mujeres y las niñas en la ciudad y 3) la reducción de todas las formas violencia, en particular de la violencia sexual en la zonas de intervención. Con esto se espera incidir en el fortalecimiento de la ciudadanía femenina y la transformación de los enfoques de seguridad en los espacios públicos.

A continuación presentaremos las acciones y los mecanismos implementados en algunas de las ciudades, tendientes a erradicar las violencias en los espacios públicos, que surgen de la adecuación del programa a los contextos específicos de cada ciudad $\mathrm{y}$ de los factores que producen mayor inseguridad y han sido evidenciados en estudios exploratorios e investigaciones previas a su implementación.

El programa "Ciudades sin violencia hacia las mujeres, ciudades seguras para todas y todos", promovido en 2006 por la Red Mujer y Hábitat, con el apoyo de la Oficina Regional del Fondo de Naciones Unidas para la Mujer (Unifem, hoy ONU Mujeres) en ciudades de Argentina (Rosario), Brasil, Colombia (Bogotá), Chile (Santiago), El Salvador, Guatemala y Perú, se constituye en un importante antecedente de las decisiones políticas y administrativas para construir un entorno amigable con y para las mujeres. En su desarrollo, la seguridad urbana se constituyó en una dimensión específica de análisis por su relevancia en la calidad de vida contemporánea y en la vida de las mujeres. Los resultados fueron indicativos del impacto diferencial que tiene en las mujeres la inseguridad en las ciudades, que se convierte en un condicionante para el uso del espacio público. Durante su desarrollo se crearon campañas pioneras que hicieron visible la violencia sexual que sufren las mujeres en el transporte público y acciones preventivas para superar la violencia de género en los espacios públicos (Red Mujer y Hábitat de América Latina, 2011).

Asimismo, en la ciudad de Seúl, desde 2007, surgió el programa Woman Friendly City, una política pública que antecedió proyectos sobre cuidado infantil, con actividades para las mujeres con menos privilegios, como madres solteras o migrantes, la creación de empleo para la mujer, el mejoramiento de sus condiciones actuales de trabajo en la ciudad y la adecuación de la seguridad en instalaciones públicas para promover empleo a las mujeres y el equilibrio del trabajo productivo y reproductivo en el entorno de la ciudad. Entre las acciones implementadas se destaca la definición de estándares para el diseño de espacios públicos como parques y calles con enfoque de género, la disposición de taxis exclusivos para mujeres conducidos por mujeres y plataformas tecnológicas para información de rutas de transporte (ONU Mujeres, 2017a).

En la ciudad de Quito, Ecuador, se realizó en 2012 la campaña "Quiero andar tranquila, calles sin acoso", realizada por el Distrito Metropolitano de Quito, en la que se propusieron acciones para el avance del derecho a las mujeres y las niñas a tener una vida libre de violencia en las calles. Entre las acciones propuestas se encuentra un nuevo marco normativo para la erradicación de la violencia de género en los espacios públicos y el establecimiento de medias para controlar el acoso sexual callejero (Ordenanza 0235 del Concejo Metropolitano de Quito), y destaca la transversalización del enfoque de género en la gestión municipal, el fortalecimiento de las capacidades de funcionarios municipales en la atención de mujeres víctimas de violencia y la creación de un sistema eficiente de recopilación y 
procesamiento de información sobre violencias de género, planificación urbana y servicios de atención integral para víctimas.

Como producto de esta decisión político-administrativa se creó el Plan de Ciudad Segura para las mujeres en 2013 con recursos propios, el cual propuso estrategias para el diálogo entre las distintas instituciones comprometidas y la definición de componentes tales como barrios seguros para mujeres y niñas, orientados por la Comisión Intersectorial para el fortalecimiento de capacidades de líderes barriales y organizaciones de mujeres. Como resultado de su trabajo se identificaron los lugares inseguros para las mujeres y las niñas y se elaboraron planes de mejoramiento para los barrios que determinaron responsabilidades para los actores involucrados. Iniciativas como "Transporte seguro", cuyo resultado fue la declaratoria de emergencia del transporte público para incorporar medidas de visibilización y atención de la violencia y el acoso, llevaron a conformar una comisión en la que participaron las secretarías de Movilidad e Inclusión Social, la Empresa de pasajeros de Quito, la Comisión de Movilidad Metropolitana, el Patronato y ONU Mujeres.

También está la creación, en 2014, del Protocolo de actuación en casos de acoso y violencia sexual en el Sistema Metropolitano de Transporte de Pasajeros de Quito, a partir del cual se definen puntos seguros en cinco de las estaciones del transporte público masivo para atención a mujeres víctimas de violencia y se inauguran paradas planificadas y habilitadas con enfoque de género que integran sistemas de seguridad, entre ellos la aplicación de celular "Bájale al acoso", implementada en 2017 para denunciar las situaciones de acoso sexual en el transporte público mediante un mensaje SMS que Vol. 23 N. ${ }^{\circ} 2$ julio - diciembre de 2018 pp. $59-82$ alerta al conductor y la Policía Metropolitana y activa el procedimiento de emergencia para dar atención a las víctimas, además de campañas comunicacionales de sensibilización en las paradas y en los buses. Por último, el componente "Fortalecimiento de servicios municipales para prevenir y atender la violencia sexual contra las mujeres y niñas en el espacio público", tendiente a superar las situaciones donde podría ocurrir acoso, el temor de las mujeres para concurrir en esquinas o calles, parques y canchas frecuentadas asiduamente por grupos de hombres y la sensación de inseguridad de mujeres jóvenes asediadas al salir de los colegios, entre otras situaciones que motivan la terminación de actividades diarias en horario diurno (ONU Mujeres, 2015b).

En Ciudad de México (CDMX), en 2013, a través de Memorándum de Entendimiento se implementó el Programa de Ciudades Seguras cuya acción principal es el Programa para prevenir y atender la violencia y acoso sexual en el transporte público y en los recorridos que realizan las mujeres para acceder a este. Otras acciones realizadas son las campañas de prevención de la violencia en las que se involucran hombres y niños para transformar los comportamientos y las actitudes frente a las situaciones de violencia contra las mujeres y las niñas, la aplicación móvil que brinda información y facilita la denuncia de incidentes o riesgos y el sistema de monitoreo integral para el transporte público. Previamente, fueron implementadas acciones como el programa Viaja Segura en 2007, en el marco del cual se realizaron campañas de difusión, destinación de los primeros vagones exclusivos para mujeres en horas con mayor afluencia de los sistemas de transporte Metro y Metrobús y la creación de módulos de atención para interponer denuncias o el uso de la palanca de seguridad para dar aviso. Además, se implementó la plataforma tecnológica Vive Segura, 
mediante la cual se ubican zonas de riesgo y se reportan en línea situaciones de violencia sexual, tales como silbidos, miradas lascivas, propuestas sexuales, violación, comentarios indecentes, obstrucción de la circulación, persecución, fotografías y videos sin consentimiento, exhibición de órganos sexuales y abuso sexual, como tocamientos y manoseo. En la actualidad, el Programa Ciudades Seguras, desde 2016, se encuentra en fase de estudio en las ciudades de Puebla y Torreón (ONU Mujeres, 2017b).

En 2013, la ciudad del Cairo, en Egipto, fue pionera del programa en los barrios menos favorecidos, como Manshiet Nasser, Ezbet El Haggana e Imbaba, donde se implementaron infraestructuras que mejoraron la seguridad de las mujeres y se promovieron campañas para el cambio de actitudes características de una cultura del acoso y el abuso sexual. Además, su Ministerio de Vivienda, Servicios y Desarrollo Urbano promovió caminatas exploratorias en las que participaron hombres y mujeres, quienes aportaron ideas y propuestas para orientar la planificación urbana, particularmente el diseño de escuelas y otros sitios donde se pretendió promover la seguridad en el espacio público y relaciones de género respetuosas (Cooperación Española AECID, 2014). Otras acciones pioneras se desarrollaron en 2010 en Kigali, Ruanda, tendientes a lograr un transporte público seguro, como la formación para los conductores de autobuses y los moto-taxis, medios de transporte principales en la ciudad, y la creación del concurso Sing yes to Kigali Safe City for Women and Girls, que invitó a músicos locales a componer canciones con mensajes que concientizaran sobre las consecuencias del abuso y promovieran la detención del acoso sexual contra mujeres y niñas, las cuales fueron ampliamente difundidas (ONU Mujeres, 2015a). En Nueva Delhi, India, en el mismo año, se instalaron circuitos cerrados de televisión (CCTV) en los buses urbanos y GPS en los taxis para realizar el seguimiento al transporte público usado diariamente por mujeres y niñas; además se tomaron medidas para iluminar correctamente las rutas que conducen hacia escuelas y universidades porque se consideraban peligrosas (ONU Mujeres, 2015a).

Desde 2011 en Port Moresby, Papúa Nueva Guinea, fueron intervenidos los mercados informales donde las mujeres estaban expuestas a múltiples violencias como compradoras y vendedoras: en dichos lugares sufrían extorsiones frecuentes y eran obligadas a abandonar el sitio en el que se encontraban desarrollando su actividad para ubicarse en lugares alejados e inseguros, donde eran víctimas de situaciones de violencia física, sexual y patrimonial. La implementación del programa facilitó una intervención en los mercados que incluyó infraestructura para mejorar la situación de salubridad en los espacios, tales como baños públicos, un sistema de mejoramiento de los acueductos de aguas residuales y el acceso a agua potable. Igualmente, promovió la creación de la asociación de vendedores y vendedoras, en la cual se cuenta con una representación de $50 \%$ de mujeres para que trabajen unidos por mejorar las condiciones generales en los mercados y mantengan la salubridad y la seguridad. Otras acciones fueron el establecimiento de un sistema de pago mediante teléfonos móviles para evitar las extorsiones y los hurtos, la adecuación de espacios para la recreación donde las hijas y los hijos de las vendedoras pueden jugar y estudiar mientras sus madres trabajan, además de puntos de información y denuncia de situaciones de acoso y violencia. Finalmente, en la ciudad de Río de Janeiro, Brasil, se han implementado acciones tecnológicas tales como clicque180, una aplicación y página web que informa sobre la legislación existente, permite la denuncia de casos de agresión 
y difunde las rutas de atención que informan qué hacer en casos de violencia; además, cuenta con un protocolo para reportar lugares inseguros con el fin de georreferenciarlos y reportar los feminicidios (ONU Mujeres, 2015a).

Estas propuestas han tenido como sustento el enfoque de seguridad humana propuesto por la Organización de las Naciones Unidas a finales de los ochenta y principios de los noventa, a partir del cual se considera la inseguridad como resultado de la insatisfacción de las necesidades básicas de los individuos por diferentes causas posibles, como la degradación medioambiental, los desastres naturales, el acelerado crecimiento demográfico y la violencia sociopolítica, entre otras, que deben ser satisfechas por el Estado para garantizar la seguridad de la población. Dicho enfoque aporta al desafío de crear ciudades seguras para las mujeres, cuyo reto es superar la violencia urbana para que las mujeres puedan percibir las urbes, vivenciarlas y realizar transformaciones profundas en las relaciones de género (Red Mujer y Hábitat de América Latina, 2011).

\section{La lucha de las mujeres por la transformación de las ciudades}

La lucha de las mujeres por alcanzar una igualdad real de oportunidades, ejercer sus derechos e incidir en la vida pública local y nacional las convierte en protagonistas en los debates globales acerca de los derechos emergentes, entendidos como una nueva comprensión de los derechos humanos reconocidos o como nuevos derechos reclamados por la sociedad civil en respuesta a los cambios y las transformaciones globales. Los avances institucionales por posicionar un programa mundial de ciudades seguras para las mujeres es producto de su movilización en los pequeños espacios y su capacidad de agencia en escenarios internacionales, en los que han exigido la intervención de los Estados con fundamento en el conocimiento producido acerca de la realidad de las mujeres en el contexto urbano. Reclamar una ciudad segura para las mujeres les ha significado exigir su derecho a la ciudad ${ }^{5}$, concepto acuñado por Henri Lefebvre (1978) en 1968, que hoy leemos con lentes de género para exigir ciudades en las que los derechos humanos de las mujeres sean reconocidos con el avance en la configuración y el diseño de ciudades inclusivas. Como señala Falú (2016), "las mujeres le han dado a la vida en la ciudad nuevos significados, no estáticos, transformándola, y han sumado a ese enjambre vital nuevos contenidos que ponen en conflicto y en cuestión, los parámetros tradicionales de cómo son vividas las ciudades, para quienes están organizadas, a quienes benefician y a quienes desplazan" (p. 18).

Las miradas feministas a los problemas sociales producidos por la inseguridad urbana, aportadas por los colectivos urbanos y los movimientos sociales de mujeres y feminista en América La-

5 La demanda y la reflexión sobre el derecho a la ciudad se enmarca en las discusiones internacionales iniciadas desde la Cumbre de la Tierra realizada en Río de Janeiro (Brasil) en 1992, en la cual se redactó el Tratado sobre Urbanización "Por ciudades, villas y poblados justos, democráticos y sustentables"; la Cumbre de la Tierra, Túnez, 1992; el Foro Internacional sobre Medio Ambiente, Pobreza y Derecho a la Ciudad; y la Carta Mundial por el Derecho a la Ciudad construida por delegados de 35 países en la Asamblea Mundial de Pobladores "Repensando la ciudad desde la gente", realizada en México en el año 2000, retomada con posterioridad en 2003 y 2005 en el III y V Foro Social Mundial en Porto Alegre (Brasil). Todos estos antecedentes son un mosaico de propuestas orientadas a la construcción de ciudades democráticas, incluyentes, educadoras, habitables, sustentables, productivas y seguras. Ha sido el resultado de la lucha de movimientos populares, organizaciones no gubernamentales, asociaciones profesionales, foros y redes nacionales e internacionales de la sociedad civil comprometidos con las luchas sociales por ciudades justas, democráticas, humanas y sustentables, y el desafío de una vida urbana sustentable basada en los principios de solidaridad, libertad, equidad, dignidad y justicia social (Carta Mundial por el Derecho a la Ciudad, 2013). 
tina, han producido múltiples críticas referidas a los modelos de intervención y las estructuras tradicionales sobre las cuales se ha gestionado la seguridad ciudadana y la seguridad pública por desconocer la violencia sistémica que experimentan las mujeres. Las mujeres han participado de este debate global y convierten la defensa del derecho a la ciudad en una lucha política: "tamañas conquistas no pueden ni deben convertirse en retórica, no obstante, corren el riesgo de ser solo papeles si no se logra una verdadera comprensión y aprehensión de sus contenidos" (Falú, 2016, p. 16). En la actualidad, los planteamientos feministas se encuentran recogidos en distintos documentos de soft law ${ }^{6}$ que, sin ser vinculantes ni producir efectos jurídicos, tienen relevancia jurídica y política por existir un relativo consenso internacional sobre su contenido y representar la voz de las mujeres que exigen mejores condiciones de vida para ellas en las urbes.

En defensa del derecho a la ciudad y el logro de ciudades seguras, el feminismo avanza en la defi-

$6 \quad$ Entre ellos se encuentra La Carta Mundial por el Derecho a la Ciudad debatida en el Foro Social de Porto Alegre en 2005, La Carta Europea de la Mujer en 1995, presentada en la II Conferencia de Naciones Unidas sobre Asentamientos Humanos Hábitat II en Estambul en 1996, en la que identificaron aspectos prioritarios para su vida en la ciudad tales como el planeamiento urbano y el desarrollo sostenible, la seguridad, la movilidad, el hábitat y los equipamientos locales, y la definición de estrategias urbanas que garanticen a las mujeres su acceso al empleo, la proximidad y la calidad de los servicios comunitarios para el cuidado, el acceso y la participación en los centros de decisiones, el acceso a la cultura y a las actividades de ocio, la seguridad y las medidas contra todos los factores de inseguridad en las ciudades, el equilibrio en el transporte individual y colectivo y la calidad y conservación del medio ambiente. Otros son la Carta Europea para la igualdad de mujeres y hombres en la vida local (20052006), la Carta Internacional por el derecho de la mujer a la ciudad de Barcelona (2004), la Declaración de Aguascalientes-México, Las ciudades desde los derechos de las mujeres (2013), y recientemente la Declaración de Quito sobre Ciudades y Asentamientos Humanos Sostenibles para Todos y la nueva Agenda Urbana producida en la Conferencia Hábitat III. nición de principios de planificación urbana, entre ellos saber dónde se está y a dónde se va (principio de señalización) para transformar sectores que no cuentan con suficiente nomenclatura y señalización, tienen deficiente iluminación o no tienen los accesos peatonales. El principio "ver y ser visto" (principio de visibilidad) a partir del cual se identifica que los espacios verdes vacíos, los baldíos y los callejones son sitios peligrosos para ellas. Los principios "oír y ser oído" (principio de concurrencia de personas) y poder obtener auxilio o escapar (principio vigilancia formal o acceso a la ayuda) permite a las mujeres contar con la posibilidad en el territorio de contar con ayuda en situaciones de peligro y agresión, donde se considera como un factor de riesgo no contar con teléfonos públicos disponibles o sistemas de alerta; el principio "vivir en un ambiente amplio y acogedor" (principio de planificación y mantenimiento de lugares) se dirige a superar aspectos como el mal manejo de los residuos que hacen algunas personas de la comunidad y las irregularidades en la recolección de basuras, por ser situaciones que afectan su disfrute del entorno y lo vuelven inseguro. Finalmente, el principio "actuar en conjunto" (participación de la comunidad) valora la importancia del disfrute de una ciudadanía plena de las mujeres. Estos principios han sido parte de las metodologías propuestas por la Red Mujer y Hábitat de América Latina, expresión del movimiento feminista y espacio de producción y reflexión feminista sobre las ciudades latinoamericanas, en cuyas investigaciones pioneras en los temas de ciudades seguras o ciudades libres de violencia para las mujeres identificaron los factores de riesgo existentes en las ciudades para las mujeres, lo que representa un aporte del feminismo para la planificación de las urbes (Red Mujer y Hábitat de América Latina, 2011). Por último, la utilización de la categoría de género para analizar el modo de vida urbano y las 
realidades urbanas nos ha conducido a concluir que las ciudades no son neutras y que en estas se configuran identidades de género, argumento con el que se explica que las diferencias entre hombres y mujeres relativizan la inseguridad a las que ellas y otros grupos socialmente discriminados se encuentran expuestos por la imposición del poder masculino hegemónico en la sociedad.

\section{Conclusiones}

Las violencias contra las mujeres en los espacios urbanos se expresan de múltiples formas, las cuales son constitutivas o no de delitos y que, en gran parte, son aceptadas y naturalizadas por las mujeres y la sociedad en general, quienes las asumen como pautas de intercambio cotidiano en los espacios públicos, las calles, los parques, las plazas y los establecimientos públicos que, a su vez, son epicentros de conductas delictivas. A pesar de los avances, las situaciones de violencia sexual callejera no han sido suficientemente investigadas ni incluidas en los procesos de planeación y planificación urbana, por lo que las iniciativas realizadas en el marco del Programa Safe Cities en el mundo constituyen experiencias importantes para ser implementadas en otros contextos urbanos.
Particularmente, en la ciudad de Medellín la violencia sexual callejera y la violencia urbana imponen a las mujeres limitaciones al ejercicio de su ciudadanía plena y al disfrute en igualdad de oportunidades de sus derechos humanos en las urbes. Además, limitan su disfrute de la ciudad como un derecho, lo cual produce consecuencias como su poca participación en la vida pública, la restricción de sus libertades y el detrimento en sus condiciones de vida o sentimientos de temor y zozobra que les impiden vivir una vida plena en igualdad de condiciones respecto a los hombres. En la actualidad, los avances promovidos mediante acciones y programas estratégicos requieren mantener su continuidad y fortalecerse política, técnica y presupuestalmente, además de ser complementados con el desarrollo de innovadoras estrategias para intervenir en los espacios públicos, las calles y los medios de transporte. Asimismo, es prioritario el diseño de modelos de sensibilización y educación para transformar la cultura machista y sexista que naturaliza la violencia callejera como un asunto cotidiano. Por último, las ideas acá expuestas contribuyen a pensar otros contextos urbanos que podrán ser aplicables en pro de lograr cada vez más ciudades donde la seguridad de las mujeres, la transformación de las relaciones entre los géneros, el derecho al goce de la ciudad y la garantía de sus derechos humanos sean asuntos prioritarios. 


\section{Referencias}

samblea General de las Naciones Unidas. (1979). Convención sobre la Eliminación de todas las Formas de Discriminación Contra la Mujer (CEDAW). Recuperado de https://www.ohchr.org/sp/professionalinterest/pages/cedaw.aspx.

Carta Mundial por el Derecho a la Ciudad. (2013). Cuadernos Geográficos, 52, 368380. Recuperado de http://www.redalyc.org/articulo.oa?id=17128112016.

Colombara, M. (2011). Violencia urbana, su relación con la violencia de género. Revista Geográfica de América Central, 2(47E). Recuperado de http://www.revistas. una.ac.cr/index.php/geografica/article/view/2861.

Concejo de Medellín. (2003). Política Pública para las Mujeres Urbanas y Rurales (Acuerdo 22 de 2003). Recuperado de https://www.medellin.gov.co/irj/go/km/ docs/wpccontent/Sites/Subportal\%20del\%20Ciudadano/Equidad\%20de\%20 G\%C3\%A9nero/Secciones/Normas/Documentos/2011/Acuerdo\%2022de\%20 2003Pol\%C3\%ADtica\%20Pca\%20\%20Mujeres\%20Urb\%20y\%20Rurales.pdf.

Concejo de Medellín. (2007). Acuerdo 01 de 2007. Por el cual se crea la Secretaría de las Mujeres y se modifica el Decreto Municipal 151 de 2002. Recuperado de https://www.medellin.gov.co/irj/go/km/docs/wpccontent/Sites/Subportal\%20 del\%20Ciudadano/Equidad\%20de\%20G\%C3\%A9nero/Secciones/Normas/ Documentos/2011/Acuerd.Mcpal\%2001_2007Modif\%20Dec.Mcpal\%20 151_2002\%20FIRMAS.pdf.

Concejo de Medellín. (2011). Acuerdo 52 de 2011. Programa de Protección Integral a Mujeres Víctimas de Violencias. Recuperado de https://www.medellin.gov.co/ irj/go/km/docs/wpccontent/Sites/Subportal\%20del\%20Ciudadano/Equidad\%20 de\%20G\%C3\%A9nero/Secciones/Normas/Documentos/2011/Acuerdo\%20 52\%20de\%202011.pdf.

Convención Interamericana para prevenir, sancionar, y erradicar la violencia contra la mujer "Convención de Belém do Pará". (1994). Recuperado de http://www.oas. org/juridico/spanish/tratados/a-61.html.

Cooperación Española AECID. (6 de marzo de 2014). Ciudades Seguras - EI Cairo. [Archivo de video]. Recuperado de https://www.youtube.com/ watch?v=uSU7BOyqF_o.

Corporación para la Vida Mujeres que Crean y Corporación Vamos Mujer. (2015). Las mujeres en los medios: estereotipo y cosificación. XIII Informe de la situación de violación de derechos humanos de las mujeres en Medellín, 2014. Medellín: Corporación para la Vida Mujeres que Crean y Corporación Vamos Mujer.

Del Valle, T. (1997). Andamios para una nueva ciudad. Lecturas desde la antropología. Madrid: Ediciones Cátedra, Universidad de Valencia, Instituto de la Mujer.

Departamento Administrativo Nacional de Estadística (2014). Encuesta nacional de calidad de vida (ECV) 2014. Recuperado de https://www.dane.gov.co/index.php/ estadisticas-por-tema/salud/calidad-de-vida-ecv/encuesta-nacional-de-calidad-devida-2014. 
Falú, A. (2016). Mujeres y ciudades. En Pra Lá e Pra cá. El derecho de las mujeres a las ciudades (pp. 15-29). Brasilia: Rede de Desenvolvimiento Humano (Redeh). Recuperado de http://www.pralaepraca.org.br/PraLaEPraCa.pdf.

Falú, A. y Rainero, L. (Eds.). (1995). Hábitat urbano, una visión de género. Córdoba: Ciscsa.

Harding, S. (1996). Feminismo y Ciencia. Barcelona: Morata.

Hernández Sampieri, R., Fernández Collado, C. y Baptista Lucio, P. (1991). Metodología de la investigación. México, D. F.: McGraw-Hill.

Jacobs, J. (2011). Muerte y Vida de las Grandes Ciudades. España: Capitán Swing Libros.

Lefebvre, H. (1968). El Derecho a La Ciudad. España: Capitán Swing Libros.

Mackinnon, C. (1995). Hacia una teoría feminista del Estado. Madrid: Ediciones Cátedra, Universidad de Valencia, Instituto de la Mujer.

Montoya Ruiz, A. M. y Builes Ceballos, Á. M. (2015a). Estudio Exploratorio de la Comuna 3. Manrique, de la Zona Nororiental de Medellín, para la implementación del Programa Ciudades Seguras Sin Violencia Hacia las Mujeres y Niñas ONU Mujeres, Fase Uno. [Informe técnico]. Medellín: ONU Mujeres, Secretaría de las Mujeres, Alcaldía de Medellín.

Montoya Ruiz, A. M., \& Builes Ceballos, Á. M. (2015b). Estudio Exploratorio de la Comuna 3, Manrique, de la Zona Nororiental de Medellín, para la implementación del Programa Ciudades Seguras Sin Violencia Hacia las Mujeres y Niñas ONU Mujeres, Fase Dos. [Informe técnico]. Medellín: ONU Mujeres, Secretaría de las Mujeres Alcaldía de Medellín.

Montoya Ruiz, A. M. y Sierra Camargo, J. (2013). Análisis de decisiones judiciales en Colombia en casos de mujeres víctimas de violencia intrafamiliar (violencia contra la pareja). Estudio de casos en la ciudad de Cartagena. En B. Londoño Toro (ed.), El papel de los jueces contra la violencia de pareja en Colombia 2005-2009 (pp. 127178). Bogotá: Universidad del Rosario.

ONU Hábitat. (2017). UN Habitat Global Activities Report 2017 Strengthening Partnerships in Support of the New Urban Agenda and the Sustainable Development Goals. Kenia: United Nations Human Settlements Programme (UN-Habitat).

ONU Mujeres. (2013). Hechos y cifras: acabar con la violencia contra mujeres y niñas. Recuperado de http://www.unwomen.org/what-we-do/ending-violence-againstwomen/facts-and-figures.

ONU Mujeres. (2015a). Proceedings Report Safe Cities Global Leader's Forum. Recuperado de http://www.unwomen.org/-/media/headquarters/attachments/ sections/library/publications/2015/un\%20women_safe_cities_global_leaders_ forum_report_final.pdf?la $=$ en\&vs $=3740$

ONU Mujeres. (2015b). Webconferencia: Ciudades seguras para mujeres y niñas y Análisis de datos de Latinobarómetro sobre victimización. Ciclo de webconferencias Seguridad, Desarrollo Humano y Prevención de las Violencias en América Latina. Recuperado de http://lac.unwomen.org/es/noticias-y-eventos/articulos/2015/08/ webconferencias. 
ONU Mujeres. (2017a). Institucionalice un programa de ciudades seguras para las mujeres dentro de la comunidad. Recuperado de http://www.endvawnow.org/es/ articles/277-institucionalice-un-programa-de-ciudades-seguras-para-las-mujeresdentro-de-la-comunidad.html

ONU Mujeres. (2017b). Programa insignia ciudades y espacios públicos seguros para mujeres y niñas. Recuperado de https://issuu.com/onumujeresmexico5050/docs/ ciudades_seguras18feb.

ONU Mujeres. (s. f.). Crear espacios públicos seguros. Recuperado de http://www. unwomen.org/es/what-we-do/ending-violence-against-women/creating-safepublic-spaces

Red Mujer y Hábitat de América Latina. (2011). Construyendo ciudades seguras: experiencias de redes de mujeres en América Latina. [Programa Regional "Ciudades sin violencia hacia las mujeres, ciudades seguras para todas y todos"]. Santiago de Chile: Red Mujer y Hábitat de América Latina, Ediciones Sur.

Rubin, G. (1986). El tráfico de mujeres: notas sobre la economía política del sexo. Nueva Antropología, 30, 95-145.

Scott, J. W. (1986). El género: una categoría útil para el análisis historico. Historical Review, 91, 1053-1075.

Secretaría de Seguridad y Convivencia. (2017). Cifras sobre violencias contra las mujeres en la ciudad de Medellín CAVIF de la Fiscalía General de la Nación - Seccional Medellín - $1^{\circ}$ de enero a 31 de diciembre 2016. Medellín: Alcaldía de Medellín. 